\title{
Biomarcadores cardiacos como ayuda diagnóstica en las cardiopatías
}

\author{
Ricardo A. Correa-Salgado*, M.Sc. ${ }_{1}$, Santiago Galvis-Arango, $\mathrm{MVZ}_{1}$
}

${ }^{1}$ Facultad de Ciencias Agropecuarias, Universidad de Caldas, Manizales, Colombia

Recibido: 18 de febrero del 2014. Aprobado: 30 de mayo del 2014.

*Autor de correspondencia: Ricardo A. Correa-Salgado. Universidad de Caldas, Manizales, Caldas, calle 65 n. 26-10. Teléfono: (+57) 3006529835

Correo electrónico: ricardo.correa@ucaldas.edu.co

Cómo citar este artículo: Correa-Salgado RA, Galvis-Arango S. Biomarcadores cardiacos como ayuda diagnóstica en las cardiopatías. Spei Domus. 2014;10(20):41-48. doi: http://dx.doi.org/10.16925/sp.v10i20.885

Resumen. En la clínica diaria de pequeños animales, es común encontrarse con patologías cardiacas, para cuyo diagnóstico se utilizan técnicas imagenológicas como la radiografía, el electrocardiograma y la ecocardiografía. Sin embargo, a pesar de la amplia investigación sobre estas técnicas, todavía es difícil establecer con claridad el pronóstico de estos pacientes y evaluar objetivamente la evolución de dichas patologías; incluso, existen pacientes con cardiopatías ocultas que pueden pasar inadvertidas al examen clínico y a las ayudas imagenológicas. Es por este motivo que en los últimos años se vienen estudiando nuevos métodos para diagnosticar cardiopatías de forma rápida y eficaz; es el caso de los biomarcadores cardiacos, como las troponinas y los péptidos natriuréticos, que en medicina humana y animal se usan cada vez más para ayudar a establecer el diagnóstico, el pronóstico y la evolución de problemas cardiacos, con alta especificidad y sensibilidad. El presente artículo pretende actualizar los conceptos sobre el manejo de biomarcadores cardiacos en el diagnóstico cardiológico de perros y gatos.

Palabras clave: caninos, enfermedad cardiaca, péptido natriurético, troponina.

\section{Cardiac Biomarkers to Help Diagnose Cardiopathies}

Abstract. In the daily clinic of small animals, it is very common to find cardiac pathologies, which are diagnosed using imageology examinations like radiology, electrocardiograms and echocardiography. However, in spite of the broad researches on such techniques, it is still hard to clearly make the patient's prognosis and objectively evaluate such pathologies. There are even patients with hidden heart diseases that may not be observed in the clinical and imageology examinations. Therefore, new methods have been studied in the past years to quickly and effectively diagnose heart diseases. Such is the case of cardiac biomarkers, like troponins and natriuretic peptides, which are more frequently used in human and animal medicine to help make the diagnosis, prognosis and evolution of heart issues in a very precisely and specifically manner. This article is intended to update the concepts on cardiac biomarkers in heart diagnosis of cats and dogs.

Keyword: canines, heart disease, natriuretic peptide, troponin.

\section{Biomarcadores cardíacos como ajuda} diagnóstica nas cardiopatias

Resumo. Na clínica diária de pequenos animais é habitual char patologias cardíacas, que para seu são empregadas técnicas de imagenologia tais como a radiografia, o eletrocardiograma e a ecografia. No entanto, pese a ampla pesquisa sobre estas técnicas, ainda é difícil estabelecer claramente o prognostico destes pacientes e avaliar objetivamente a evolução de tais patologias; incluso, há pacientes com cardiopatias ocultas que podem passar inadvertidas ao teste clínico e as ajudas da imagenologia. É por isso que nos últimos anos novos métodos para diagnosticar cardiopatias rápida e eficazmente estão sendo estudados; é o caso dos Biomarcadores cardíacos, como as troponinas e os peptídeos natriuréticos, que em medicina humana e animal são usados a cada vez mais para ajudar no estabelecimento de diagnostico, o prognostico e a evolução de problemas cardíacos, com alta especificidade e sensibilidade. Este artigo pretende atualizar os conceitos sobre o manejo de biomarcadores cardíacos no diagnóstico cardiológico de cães e gatos.

Palavras-chave: caninos, doença cardíaca, peptídeo natriurético, troponina. 


\section{Introducción}

Las enfermedades cardiovasculares son comunes en caninos y felinos, y a pesar de un minucioso examen clínico y de pruebas diagnósticas como la radiografía, el electrocardiograma, la ecocardiografía y la medición de la presión arterial, todavía es difícil estimar la gravedad y la evolución de dichas patologías [1].

Durante la última década, una nueva generación de biomarcadores cardiacos y neurohormonales se han desarrollado en medicina humana para el diagnóstico de infarto agudo de miocardio, y estudios recientes empiezan a mostrar su utilidad en el diagnóstico de diferentes patologías cardiacas en animales [2].

Un biomarcador se define como una sustancia elaborada por un tejido específico que puede ser detectado en la circulación. Generalmente, se trata de una proteína que, medida en sangre, refleja la presencia y la severidad de una enfermedad o los efectos de un tratamiento sobre esta [3]. Para que sea útil desde el punto de vista clínico, debe liberarse en una cantidad proporcional a la evolución del proceso patológico y proporcionar información sobre la presencia, la severidad y el pronóstico de la enfermedad [4].

Algunos órganos, como el hígado y los riñones, tienen biomarcadores que permiten al clínico evaluar la función o integridad de dichos órganos (ALT, AST, creatinina, NUS) mediante su medición, y proporcionan un método rápido y poco invasivo de diagnóstico y monitoreo [5]. Hasta hace poco, el corazón carecía de pruebas similares medibles en sangre, y sólo en medicina humana se usaban la creatinkinasa y el lactato deshidrogenasa para el diagnóstico de infarto agudo de miocardio, pero dichas pruebas carecen de suficiente especificidad y sensibilidad para ser usadas en pequeños animales [2].

\section{Detección de un biomarcador cardiaco}

Las principales células del corazón son los cardiomiocitos, que se contraen y se relajan para producir un bombeo de la sangre a todo del organismo. Estas células no pueden ser regeneradas, por lo que las probabilidades de ser vulneradas son altas [6].

Cuando las células mueren, las proteínas intracitoplasmáticas salen de las células más rápidamente que las proteínas de las membranas o que los elementos fijos de la célula [6]. Este proceso ocurre igual cuando existe necrosis o hay daño celular importante en el cardiomiocito, con lo que se pierde la integridad de la membrana y se permite el paso de macromoléculas al tejido intersticial, desde donde son absorbidas por los capilares o por el sistema linfático y alcanzan finalmente la circulación sistémica. Estas macromoléculas liberadas son las que se pueden usar como marcadores biológicos del daño miocárdico, y en la actualidad son consideradas de gran relevancia en la práctica clínica, dado que pueden ser identificadas y medidas en la sangre periférica [7].

\section{Características de un biomarcador}

Para que un biomarcador tenga relevancia clínica, debe cumplir con varios aspectos importantes: debe ser específico para una enfermedad o un órgano en particular; con alta sensibilidad para que incluso niveles medibles muy bajos revelen lesión orgánica leve; con alta especificidad, es decir que no se liberen de otros órganos o tejidos distintos al de interés; con costos bajos, y con procesos sencillos de realizar y que tengan relevancia clínica [6], [8], [9].

El interés clínico de la utilización de biomarcadores no sólo radica en ser una forma de rápido diagnóstico de cardiopatías, sino que también nos da la oportunidad de estratificar la enfermedad y de evaluar realmente el impacto de los tratamientos instaurados en el paciente, además de establecer un pronóstico [10].

\section{Tipos de biomarcadores cardiacos}

Los marcadores cardiacos incluyen cuatro tipos, relacionados con distintas condiciones clínicas, como se expone a continuación:

1. Troponinas cardiacas, que se liberan en necrosis o lesión del miocardio. Son relevantes en veterinaria la troponina $\mathrm{L}$ ( $\mathrm{TnI}$ ) y la troponina $\mathrm{T}$ ( $\mathrm{TnT}$ ).

2. Péptidos natriuréticos, que evalúan la función miocárdica y la sobrecarga de volumen auricular. Se evalúa en veterinaria el péptido natriurético auricular (ANP) y el péptido natriurético tipo в (BNP) [9], [11].

3. Las lipoproteínas séricas HDL y LDL, que evalúan la homeostasis de las grasas. Aunque no son un biomarcador cardiaco propiamente dicho, están altamente relacionadas con el desarrollo de ateroesclerosis coronaria, patología de alta relevancia en medicina humana [12]. 
4. La proteína $\mathrm{C}$ reactiva $(\mathrm{PCR})$, que evalúa inflamación del sistema cardiovascular, usada en el diagnóstico y pronóstico del infarto agudo de miocardio en humanos [13].

En medicina veterinaria, las dos últimas han mostrado poca utilidad; sin embargo, a continuación, evaluaremos más a fondo los biomarcadores que, en la actualidad, se considera que aportan información importante en enfermedades cardiacas en perros y gatos cuando aún son asintomáticas, y que permiten evaluar su gravedad.

\section{Troponinas cardiacas}

La troponina (Tn) es un componente fundamental del músculo cardiaco y se libera en respuesta al daño miocárdico [14]. A nivel molecular, la troponina consta de tres subunidades (cTnI, cTnт у стnC), que ayudan a regular el acoplamiento excitación-contracción en el miocito cardiaco. La subunidad cTnI es el componente inhibidor que impide la interacción entre la actina y la miosina hasta que la subunidad стпс se una a los iones de calcio $[15,4]$.

Una alteración en los sarcómeros provoca la separación de la subunidad crni de la actina, y la consecuente rotura de la membrana celular permitirá el paso de la molécula a la circulación general. Un aumento de la cTni en suero o plasma es un indicador muy sensible y específico de daño celular miocárdico y de necrosis. En medicina veterinaria, se está aprovechando la gran homología de estas moléculas entre el perro y el humano, a fin de utilizar inmunoensayos desarrollados para este último [4].

Únicamente la troponina $\mathrm{T}$ y la troponina I tienen interés en el diagnóstico de lesiones del miocardio, ya que están codificadas por genes diferentes a los que codifican la proteína en el músculo esquelético, lo que le otorga su especificidad diagnóstica [16]. Adicionalmente, las troponinas cardiacas sólo tienen una pequeña fracción disuelta en el citoplasma de los cardiomiocitos (el $8 \%$ de $\mathrm{Tn}$ t en humanos y el $2 \%$ en perros es citosólica), por lo que cualquier elevación en sus niveles plasmáticos es sugerente de lesión miocárdica; este hecho, junto a su pequeño tamaño molecular y su especificidad tisular, otorga a esta proteína un papel adecuado en la detección temprana de lesiones celulares reversibles (isquemia) o irreversibles (necrosis) [17].
TNI

La troponina TnI es la más específica de las troponinas. Por este motivo, en ella se han centrado principalmente las investigaciones a nivel veterinario [18], y los inmunoensayos realizados para humanos se han validado en caninos [19].

La TnI es específica como biomarcador de necrosis miocárdica, pero inespecífica para determinar la causa subyacente de la lesión [4], [20], y su aumento es directamente proporcional a la severidad del daño del miocardio [21]. Se ha determinado qué patologías extracardiacas que pueden producir un aumento notable de la Tni en perros, posiblemente debido a lesión cardiaca secundaria a hipoxia o toxicosis, incluyen la piómetra, la parvovirosis [22], el síndrome de dilatación y el vólvulo gástrico (DTG), el derrame pericárdico [23], los traumatismos y las septicemias. Las miocarditis asociadas a babesia o tripanosoma [4], así como los perros positivos a Bartonella spp [24], pueden tener un aumento de hasta 100 veces su valor; igualmente, se han detectado incrementos en los niveles de TnI en perros sanos sometidos a anestesia general [25].

En un estudio en perros, se determinó que la valoración de la Tni para la evaluación de síndrome coronario provocado de manera experimental presentó $100 \%$ de sensibilidad y casi $100 \%$ de especificidad [26].

Los niveles de TnI considerados normales para perros se encuentran entre 0,03 y $0,07 \mathrm{ng} / \mathrm{ml}$ [8], y su aumento progresivo en mediciones seriadas está asociado a evolución desfavorable a largo plazo y es un factor predictor de mortalidad más confiable que detectar una elevación en el nivel de TnI en una medición aislada [27], [28].

En un estudio realizado en 120 perros, se demostró que los pacientes con niveles menores o iguales a $0,15 \mathrm{ng} / \mathrm{ml}$ eran animales jóvenes sanos, y perros con valores mayores a $1,01 \mathrm{ng} / \mathrm{ml}$ tenían enfermedades cardiacas graves [1].

\section{TnT}

Tiene una vida media corta de unos 90 minutos, y una persistente elevación en los días 3 y 4 refleja la degradación de los elementos contráctiles, que es una característica de la lesión celular irreversible [29].

Los niveles de тnT superiores a $0,05 \mathrm{ug} / \mathrm{L}$ son considerados indicativos de enfermedad cardiaca [30]. 


\section{Modo de muestreo de las troponinas}

El muestreo para la evaluación de troponinas puede ser venoso o arterial. Se puede hacer la prueba en plasma, suero o sangre total, cuyo tiempo oscila alrededor de los 75 minutos, de los cuales 15 minutos corresponden a la centrifugación, aunque ya se han desarrollado kits rápidos, que tardan 20 minutos en multiparámetros automatizados [31].

La mayoría de las pruebas contienen dos anticuerpos específicos dirigidos a dos sitios diferentes. El primer anticuerpo se denomina "captura", y como su nombre lo indica, captura toda la troponina cardiaca presente en el suero. El segundo anticuerpo, llamado "desarrollador", se añade después del lavado y se une a la troponina asociada al primer anticuerpo, que da una señal luminosa que se cuantifica y da el nivel de troponina como producto final [31].

Las secuencias de aminoácidos de ambas isoformas de troponinas son suficientemente diferentes $y$, por lo tanto, detectables por las pruebas de anticuerpos monoclonales [32].

\section{Péptidos natriuréticos}

Los péptidos natriuréticos son miembros de una familia de hormonas que se liberan a la circulación como consecuencia del estiramiento o de la tensión del miocardio de las aurículas y los ventrículos, respectivamente, y son responsables de la regulación de los fluidos corporales, la homeostasis y la presión sanguínea $[14,33]$.

Se han descrito seis tipos de péptidos natriuréticos, pero sólo el péptido natriurético auricular (ANP) y el péptido natriurético tipo в (BNP) son considerados de importancia diagnóstica en perros y gatos [16]. Los péptidos natriuréticos son biomarcadores importantes para el diagnóstico y pronóstico de enfermedades cardiacas que se acompañen de hipertrofia ventricular e insuficiencia cardiaca congestiva [11,34, 35].

El ANP procede principalmente de las aurículas, mientras que el BNP procede de miocitos auriculares y ventriculares. Ambos se liberan como moléculas precursoras que son escindidas por proteasas séricas que producen dos fragmentos en cantidades iguales; un fragmento C-terminal activo (conocidos como C-ANP y $\mathrm{C}-\mathrm{BNP}$ ) y un fragmento terminal inactivo (NT-proANP у NT-proBNP) [4].
El C-ANP y el C-BNP tienen una semivida muy corta y la determinación de la concentración circulante puede ser difícil. El NT-proANP y el NT-proBNP tienen semividas más prolongadas y son más estables en condiciones de laboratorio, lo que hace que su determinación sea más práctica en el ámbito clínico [4].

Hay que tener en cuenta que una parte de estos péptidos es excretada por riñón, por lo cual sus niveles circulantes podrían estar influenciados por la función renal. Se ha demostrado que perros con enfermedades renales crónicas presentan una concentración dos veces mayor de NT-proANP que perros sanos, aun en ausencia de cardiopatía [33].

El BNP parece tener un mejor rendimiento para proporcionar un diagnóstico en comparación con el ANP, razón por la que el primero ha tenido una mayor investigación [36].

\section{Péptido natriurético auricular (ANP)}

El ANP es una hormona polipeptídica compuesta por 28 aminoácidos, sintetizada y secretada por las aurículas en el corazón adulto [37]. Es el principal implicado en la regulación de la función renal y del volumen plasmático en condiciones fisiológicas. El aumento del volumen plasmático y de la presión arterial o de la sangre pulmonar, que llevan a distensión de la pared o a aumento de presión sobre el músculo auricular, conduce a niveles significativamente elevados de ANP $[34,35]$.

Infortunadamente, el ANP es extraído con rapidez de la circulación ( 2 a 5 minutos), sobre todo por unión a receptores e hidrólisis, por lo cual se evalúan clínicamente las concentraciones de NT-pro-ANP, que tiene una vida media mayor que el ANP (cerca de 10 veces), además de ser más estable en condiciones de laboratorio [37].

Debido a que la secuencia de aminoácidos del ANP está altamente conservada entre especies, se pueden usar los ensayos desarrollados para humanos en la medición de estas sustancias en perros [38], al igual que se están validando para otras especies como los equinos [39].

Los niveles considerados normales en caninos para la NT-pro-ANP son inferiores a $1000 \mathrm{fmol} / \mathrm{L} \mathrm{[4]}$.

Se debe tener en cuenta que la expresión y secreción de ANP se puede aumentar por estímulos como: endotelina 1, arginina, angiotensina II, alfa adrenérgicos, hormonas tiroideas, glucocorticoides, hormona del crecimiento, prostaglandinas, vitamina $\mathrm{D}$, trombina e hipoxia [40]. 


\section{Péptido natriurético cerebral o tipo в (BNP)}

El BNP es un péptido de 32 aminoácidos similar estructuralmente al ANP. El BNP es sintetizado como una prohormona, proBNP, y es descompuesto por una furina en las moléculas activas (BNP) e inactivas (NT-proBNP); ambas pueden estar en el miocardio y el plasma [41].

El BNP tiene una vida media mayor que el ANP (22 minutos) y la de NT-probNP es de 70 minutos ( 15 veces mayor que el BNP) [37].

El péptido natriurético tipo в es principalmente secretado por los cardiomiocitos ventriculares en respuesta al volumen y la sobrecarga de presión, con lo que promueve la natriuresis, la diuresis y la vasodilatación, acciones que se oponen a las anormalidades fisiológicas que son activadas durante la falla cardiaca [10].

El BNP evalúa la gravedad de la lesión isquémica, dado que la isquemia provoca un aumento de la tensión de la pared ventricular izquierda que lleva a un aumento en la producción y liberación de BNP, incluso en ausencia de necrosis o disfunción ventricular izquierda [42]. Esto se demostró en estudios que revelaban incrementos de los niveles de BNP después de una prueba de estrés con dobutamina en perros [23]; este péptido también se puede utilizar en el seguimiento de terapias cardiotóxicas como con la doxorrubicina [43].

Estudios en caninos han revelado que las concentraciones circulantes de BNP varían de acuerdo con el tipo de enfermedad del corazón y la falla cardiaca [44]. Por consiguiente, la medición de BNP puede tener importancia clínica en la diferenciación entre perros sanos y perros asintomáticos con enfermedad crónica valvular adquirida o cardiomiopatía dilatada [45].

La secuencia de aminoácidos de NT-proBNP difiere entre las distintas especies, por lo cual los inmunoensayos desarrollados para humanos no son confiables en animales; se debe utilizar una prueba validada para perros y gatos, respectivamente [46], algunas de las cuales ya están disponibles en el mercado [47].

Los niveles plasmáticos considerados normales son NT-probNP canino inferior a $210 \mathrm{pmol} / \mathrm{L}$ y NTproBNP felino inferior a $50 \mathrm{pmol} / \mathrm{L}$ [4].

\section{Usos de las pruebas de biomarcadores cardiacos}

\author{
Péptido natriurético auricular (ANP)
}

Uso actual: apoyo en diagnóstico de la insuficiencia cardiaca congestiva en perros.

Usos posibles:

- Apoyo en diagnóstico de la insuficiencia cardiaca congestiva en gatos.

- Monitoreo de respuesta al tratamiento.

- Proporcionar un pronóstico.

\section{Péptido natriurético tipo в (BNP)}

Uso actual: apoyo en diagnóstico, pronóstico y respuesta al tratamiento en perros y gatos cardiópatas.

Usos posibles:

- Diferenciar la disnea cardiaca de la no cardiaca en el perro.

- Apoyo en la detección de hipertensión pulmonar como complicación de un proceso respiratorio primario [48].

- Detección de miocardiopatía oculta en perros [46], [49].

\section{Troponina I (TNL)}

Uso actual: diagnóstico de infarto agudo al miocardio. Usos posibles:

- Evaluación de la severidad de enfermedades como estenosis subaórtica, cardiomiopatía, dirofilariasis y valvulopatía mitral en perros [50].

- Indicador del pronóstico en perros con cardiomiopatía.

- Monitoreo de respuesta al tratamiento.

- Evaluación del daño cardiaco debido a una enfermedad extracardiaca; por ejemplo, síndrome de dilatación y vólvulo gástrico [2], [4]. 


\section{Conclusiones}

Se evidencia que los biomarcadores cardiacos son una reciente y prometedora herramienta, sencilla y eficaz para el profesional en medicina veterinaria en el diagnóstico de las cardiopatías en pequeños animales.

A medida que avance la investigación y sean más accesibles las pruebas de los biomarcadores cardiacos, su uso será cada vez mayor en la práctica clínica, lo que hará posible evaluar parámetros más objetivos de pronóstico, evolución y, más importante aún, datos de respuesta al tratamiento, que permitan intervenciones precoces en la toma de decisiones farmacológicas en el paciente cardiópata.

Las troponinas cardiacas son de gran ayuda para evaluar la gravedad de la lesión miocárdica y están probablemente relacionadas con factores pronósticos.

La determinación de los niveles de péptidos natriuréticos puede contribuir al diagnóstico de cardiopatías, principalmente en pacientes con síntomas no muy claros de insuficiencia cardiaca o en aquellos para los que no se puede establecer con claridad si la causa de una disnea es cardiaca o pulmonar.

Es claro que, aunque los biomarcadores cardiacos se consideran una ayuda importante en el diagnóstico cardiológico, no pueden remplazar la información que aportan un examen clínico meticuloso y las ayudas imagenológicas como la ecocardiografía y la radiografía.

Se debe tener precaución al interpretar un dato aislado de niveles plasmáticos de biomarcadores cardiacos, pues existen varias patologías extracardiacas que pueden incrementar dichos niveles.

\section{Referencias}

[1] Fonfara S, Loureiro J, Swift S, James R, Cripps P, Dukes-McEwan J. Cardiac Troponin I as a Marker for Severity and Prognosis of Cardiac Disease in Dogs. Vet J. 2010);184(3):334-9.

[2] Oyama MA. Evaluation of Heart Function Using Blood-Based Test: Current Use and Future Applications. Documento presentado en el Congreso Internacional de la Asociación de Veterinarios de Animales de Compañía. 2006 may 19-21; Rimini.

[3] Mosbasheri A, Cassidy JP. Biomarkers in Veterinary Medicine: Towards Targeted, Individualized Therapies for Companion Animals. Vet J. 2010;185(1):1-3.
[4] Oyama MA, Reynolds C. Biomarkers in the diagnosis of canine heart disease. Veterinary Focus. 2008;18(3):1-6

[5] Miyazaki M, Rosenblum J, Kasahara Y, Nakagawa I, Patricelli MP. Determination of Enzymatic Source of Alanine Aminotransferase Activity in Serum from Dogs with Liver Injury. J Pharmacol and Toxicol Methods. 2009;60(3):307-15.

[6] Landenson JH. A Personal History of Markers of Myocyte Injury (Myocardial Infarction). Clin Chim Acta. 2007;381(1):3-8.

[7] Lopez-Sendo J. Troponinas y otros marcadores de daño miocardico. Mitos y realidades. Rev Esp Cardiol. 2003;56(1);9-16.

[8] Shinde SB. Relevant Cardiac Biochemical Markers. Bombay Hospital Journal. 2004;46(4).

[9] Solter PF. Clinical Biomarkers of Cardiac Injuries and Disease. ACvp/Asvcr. Annual Meetings. Savannah: IVIs; 2007. p. 10-4.

[10] Fox PR, Oyama MR. Utility of Plasma N-Terminal Pro-Brain Natriuretic Peptide (NT-proBNP) to Distinguish between Congestive Heart Failure and Non-Cardiac Causes of Acute Dyspnea in Cats. J Vet Cardiol. 2009;11(Supl 1):S51-S61.

[11] Kimmenade V, Januzzi J, Roland RJ. The Evolution of the Natriuretic Peptides - Current Applications in Human and Animal Medicine. J Vet Cardiol. 2009;11(Supl 1):S9-21.

[12] Papageorgiou N, Tousoulis D. Is HDL a Prognostic Biomarker for Coronary Atherosclerosis? Intern J Cardiol. 2014;174(3):465-7.

[13] Saunders AB, Smith BE, Fosgate G, Suchodolski JS, Steiner JM. Cardiac Troponin I and C-Reactive Protein Concentrations in Dogs with Severe Pulmonic Stenosis before and after Balloon Valvuloplasty. J Vet Cardiol. 2009;11(1):9-16.

[14] Beardow A. Veterinary Cardiology Diagnostics. Idexx Laboratories. 2008;1(2):12-5.

[15] O’brien PJ, Smith DEC, Knechtel TJ, et al. Cardiac Troponin I is a Sensitive, Specific Biomarker of Cardiac Injury in Laboratory Animals. Lab Anim. 2006;40(2):153-71.

[16] Jaffe AS, Ordoñez-llanos J. Troponinas ultrasensibles en el dolor torácico y los síndromes coronarios agudos, ¿un paso hacia adelante? Rev Esp Cardiol. 2010;63(7):763-9. 
[17] Capdevila C, Portoles M, Hernandiz A, Pallares V, Cosin J. La troponina $\mathrm{T}$ como posible marcador de daño miocárdico menor. Su aplicación en el miocardio aturdido y en la isquemia silente. Rev Esp Cardiol. 2001;54:508-91.

[18] Caro-Vadillo A. Troponinas: marcadores de lesión miocárdica en perros. Clin Vet Peq Anim. 2005;25(3):183-187.

[19] Sleeper M, Clifford CA, Laster LL. Cardiac Troponin I in the Normal Dog and Cat. J Vet Intern Med. 2001;15(5):501-3.

[20] Ventura M, Blanco LM, Tuñon J, Muñoz B, Madrigal J, Moreno JA, et al. Biomarcadores en la medicina vascular. Rev Esp Cardiol. 2009;62(6);677-88.

[21] Oyama MA, Sisson DD. Blood-Based Detection of Occult Heart Disease. En: Annual Veterinary Medical Forum, 21, 2001. Charlotte: American College of Veterinary Internal Medicine; 2003. p. 88-9.

[22] Kocaturk M, Martínez S, Eralp O, Tvarijonaviciute A. Tei Index (Myocardial Performance Index) and Cardiac Biomarkers in Dogs with Parvoviral Enteritis. Res in Vet Sci. 2012;92(1):24-9.

[23] Ljubica SK, Drajisa RT, Radomir R. Resting and Dobutamine Stress Test Induced Serum Concentrations of Brain Natriuretic Peptide in German Shepherd Dogs. Res Vet Sci. 2012;93(3):1446-53.

[24] Trafny D, Oyama M, Wormser C, Reynolds C. Cardiac Troponin-I Concentrations in Dogs with Bradyarrhytmias before and after Artificial Pacing. J Vet Cardiol. 2010;12(3):183-90.

[25] Verbiest T, Binst D, Waelbers T, Coppieters E, Polis I. Perioperative Changes in Cardiac Troponin I Concentrations in Dogs. Res Vet Sci. 2013;94(3):446-8.

[26] Caragher TE, Fernández BB, Jacobs FL, Barr LV. Evaluation of Quantitative Cardiac Biomarker Point-of-Care Testing in the Emergency Department. J Emerg Med. 2002;22(1):1-7.

[27] Healey JS, Davies RF, Smith SJ. Prognostic Use of Cardiac Troponin $\mathrm{T}$ and Troponin I in Patients with Heart Failure. Can J Cardiol. 2003;19(4):3836.

[28] Peacock WF, De Marco T, Fonarow GC. Cardiac Troponin and Outcome in Acute Heart Failure. N Engl J Med. 2008;358(20):2117-26.
[29] Al-Otaiby, Al-Amri, Al-Moghairi. The Clinical Significance of Cardiac Troponins in Medical Practice. J Saudi Heart Assoc. 2011;23(1):3-11.

[30] Cakiroglu, D. Meral, Y. Bakirel, U. Kazanci, D. Cardiac Troponin Levels in Dogs with Dilate Cardiomyopathy. Kafkas Univ Vet Fak Derg. 2009;15(1):13-7.

[31] Pruvot S, Galidie G, Bergmann JF, Mahé I. Troponin and Others Markers of Myocardial Ischemia Injury, Which Relevance in Internal Medicine? Rev Med Interne. 2006;27(3):215-26.

[32] Braunwald E, Antman EM, Beasley JW, Califf RM, Cheitlin MD, Hochman JS. ACC/AHA Guidelines for the Management of Patients with Unstable Angina and non-sT Segment Elevation Myocardial Infarction: A Report of the American College of Cardiology/American Heart Association Task Force on Practice Guidelines (Committee on the Management of Patients with Unestable Angina). J Am Coll Cardiol. 2000;36(3):970-1062.

[33] Connolly DJ, Soares RJ. Circulating Natriuretic Peptides in Cats with Heart Disease. J Vet Intern Med. 2008;22(1):96-105.

[34] Maack T. The Broad Homeostatic Role of Natriuretic Peptides. Arq Bras Endocrinol Metabol. 2006;50(2):198-207.

[35] Richards AM. Nuevos biomarcadores en la insuficiencia cardiaca; aplicaciones en el diagnóstico, pronóstico y pautas de tratamiento. Rev Esp Cardiol. 2010;63(6):635-9.

[36] Felker, GM, Petersen JW, Mark DB. Natriuretic Peptides in the Diagnosis and Management of Heart Failure. CMAJ. 2006;175(6):611-7.

[37] Bonet AL, Martínez-Dolz L. Péptidos natriuréticos en insuficiencia cardiaca. Rev Esp Cardiol. 2006;6(F):15-26.

[38] Trachsel DS, Grenacher B, Weishaupt MA, Schwarzwald CC. Plasma atrial natriuretic peptide concentrations in horse with heart disease, a pilot study. Vet J. 2012;192(2):166-70.

[39] Trachsel DS, Schwarzwald CC, Grenacher B, Weishaupt MA. Analytic Validation and Comparison of Three Commercial Immunoassays for Measurement of Plasma Atrial/A-Type Natriuretic Peptide Concentration in Horses. Res Vet Sci. 2014;96(1):180-6. 
[40] Ruskoaho H. Cardiac Hormones as Diagnostic Tools in Heart Failure. Endocr Rev. 2003;24(3):34156.

[41] Mair J, Hammerer-Lercher A, Puschendorf B. The Impact of Cardiac Natriuretic Peptide Determination on the Diagnosis and Management of Heart Failure. Clin Chem Lab Med. 2001;39:571-88.

[42] Futterman LG, Lamberg L. Novel Markers in the Acute Coronary Syndrome: BNP, IL-6, PAPP-A. Am J Crit Care. 2002;11(2):168-72.

[43] Alves de Souza RC, Camacho AA. Neurohormonal, Hemodynamic and Alectrocardiographic Evaluations of Healthy Dogs Receiving LongTerm Administration of Doxorubicin. Am J Vet Res. 2006;67(10):1779.

[44] MacDonald KA, Kettleson MD, Munro C, Kass P. Brain Natriuretic Peptide Concentration in Dogs with Heart Disease and Congestive Heart Failure. J Vet Intern Med. 2003;17(2):172-7.

[45] Oyama MA, Rush JE, Fox PR. Assessment of Serum N-Terminal Pro-B-Type Natriuretic Peptide Concentration for Differentiation of Congestive Heart Failure from Primary Respiratory Tract Disease as the Cause of Respiratory Signs in Dogs. J Am Vet Assoc. 2009;235(11):1319-25.
[46] Oyama MA, Sisson D, Solter PF. Prospective Screening for Occult Cardiomyopathy in Dogs by Measurement of Plasma Atrial Natriuretic Peptide, в-Type Natriuretic Peptide and Cardiac Troponin I Concentrations. Am J Vet Res. 2007;68(1):42-7.

[47] Haggstrom J, Hansson K, Kvart C, Pedersen H. Relationship between Different Natriuretic Peptides and Severity of Naturally Acquired Mitral Regurgitation in Dogs with Chronic Myxomatous Valve Disease. J Vet Cardiol. 2000;2(1):7-16.

[48] Kellihan H, Mackie B, Stepien R. NT-probnP, NTproANP and CTnI concentrations in dogs with precapillary pulmonary hypertension. J Vet Cardiol. 2011;13(3):171-82.

[49] Prosek R, Sisson DD, Oyama MA, Solter PF. Distinguish plasma atrial natriuretic factor, B-type natriuretic factor, endothelin and cardiac troponin I in cardiac and noncardiac dyspnea in 48 dogs using. J Vet Intern Med. 2007;21(2):238-42.

[50] Carretón E, Morchón R, Gonzáles-Miguel M, Juste M, Simón F, Montoya-Alonso JA. Utility of cardiac biomarkers during adulticide treatment of heartworm disease (Dirofilaria immitis) in dogs. Vet Parasitol. 2013;197(1-2):244-50. 